\title{
COEDUCACIÓN EN LA FORMACIÓN INICIAL DEL PROFESORADO: DE LA TRANSVERSALIDAD A LA VIVENCIA A TRAVÉS DE LA ÉTICA DEL CUIDADO
}

\author{
COEDUCATION IN THE INITIAL TRAINING OF TEACHERS: FROM \\ TRANSVERSALITY TO LIVING THROUGH THE ETHICS OF CARE
}

\author{
MARÍA GaRCía-CaNo TORRICO \\ UNIVERSIDAD DE CÓRDOBA \\ maria.garciacano@uco.es \\ Eva Hinojosa Pareja \\ UNIVERSIDAD DE CÓRDOBA \\ ehinojosa@uco.es
}

\begin{abstract}
RESUMEN
Los planes de formación inicial del profesorado en las universidades españolas siguen adoleciendo de la incorporación de contenidos y metodologías sobre coeducación. El artículo, desde una perspectiva reflexiva de la actividad docente, presenta las oportunidades que la aplicación de una metodología particular aplicada como Proyecto de Mejora en los Grados de Infantil y Primaria en la Universidad de Córdoba, potencia y posibilita la formación de los y las estudiantes en contenidos vinculados a los principios que definen la ética del cuidado de Carol Gilligan. Se identifican y describen las líneas de investigación que se han producido en el ámbito de la formación inicial del profesorado desde la coeducación y se definen los principios del modelo educativo feminista de la ética del cuidado. La descripción de la propuesta metodológica que se viene desarrollando durante dos cursos académicos (2014/15 y 2015/16) sirve de referente para justificar los aportes que dicho proceso de enseñanza-aprendizaje tiene para la formación inicial coeducativa de maestros y maestras a través de la incorporación de elementos como la interdependencia, la comunicación, la responsabilidad y el cuidado.
\end{abstract}

\section{PALABRAS CLAVE}

Coeducación; Formación del profesorado; Innovación docente; Educación superior; Ética del cuidado; Feminismo

\footnotetext{
ABSTRACT

Preservice teacher training programs in Spanish universities still suffer from the incorporation of contents and methodologies on coeducation. The paper, from a
} 
reflexive perspective of teaching, presents the opportunities of the application of a particular methodology, as improvement project in Childhood and Primary Education Degrees at the University of Córdoba, to enhance and facilitate the students' training in contents linked to the principles of the ethics of care defined by Carol Gilligan. Lines of research generated in the field of preservice teacher education from coeducation and principles of feminist educational model of the ethics of care are identified and defined. The description of the methodology that has been applied for two academic years (2014/15 and 2015/16) serves as a reference to justify the contributions of this teaching-learning process for a coeducative preservice teacher education.

\section{KEY WORDS}

Coeducation; Pre-service Teacher Education; Educational Innovation; Higher Education; Care Ethics; Feminism

$$
* \quad * \quad *
$$

\section{INTRODUCCIÓN}

De forma reiterada distintos trabajos señalan la dependencia que ostentan los planes de formación universitaria de la voluntad y sensibilidad de profesorado que a título individual incorpora contenidos o acciones feministas o coeducativas (García-Pérez et al., 2011; García, Sala, Rodríguez y Sabuco, 2013; Donoso-Vázquez y Velasco-Martínez, 2013). Todo ello, a pesar del reconocido avance que las aportaciones de los estudios feministas han supuesto en distintos escenarios de producción del conocimiento (Castells, 1998), para el desarrollo normativo a nivel mundial o el diseño de políticas públicas nacionales y locales.

El recorrido que la institución universitaria viene haciendo en este sentido arrastra toda una historia de denuncia y reivindicación por hacer visibles y presentes a las mujeres en docencia e investigación, pero también, para lograr que la igualdad de oportunidades en ámbitos como la gobernanza, el reparto de los tiempos, los itinerarios y trayectorias profesionales de las mujeres o la utilización de un lenguaje no sexista, por poner varios ejemplos, permee las prácticas cotidianas de la institución y de sus actores.

En este sentido, consideramos que el espacio universitario se encuentra en una coyuntura adecuada para el fomento de la enseñanza de género, feminista y 
coeducativa en este reciente estreno de siglo. Identificamos un conjunto de condicionantes de posibilidad susceptibles de ser retos y oportunidades para la docencia, la formación de profesionales y ciudadanos comprometidos y comprometidas y la promoción de una visión crítica y transformadora del contexto patriarcal actual, enmarcado en lo que se ha venido a llamar como un contexto de "crisis de la universidad"l (Manzano-Arrondo, 2015), cuestionador de la hegemonía y orientado hacia el cambio y la responsabilidad social (Walker y McLean, 2013).

Uno de estos factores lo situamos en el contexto de reforma universitaria de adaptación al Espacio Europeo de Educación Superior (EEES) que ha supuesto no solo el cambio de estructura de las titulaciones (paso de las Diplomaturas y Licenciaturas a los actuales Grados y Másteres), sino además, la oportunidad de revisar y debatir la presencia, organización y secuencia de materias y asignaturas que incorporen los debates de igualdad y género (Instituto de la Mujer, 2004), tal como se reglamenta en el artículo 3 del Real Decreto 1393/2007 que establece la ordenación de las enseñanzas universitarias oficiales o en el artículo 25 de la Ley Orgánica 3/2007 para la igualdad efectiva de mujeres y hombres cuando señala que:

[...] las Administraciones públicas promoverán: a) La inclusión, en los planes de estudio en que proceda, de enseñanzas en materia de igualdad entre mujeres y hombres. b) La creación de postgrados específicos. c) La realización de estudios e investigaciones especializadas en la materia.

Coherentes con la legislación nacional en materia de igualdad, las directrices del Gobierno fueron la incorporación de materias especializadas en estudios de género en todas las titulaciones de Grado, el desarrollo de posgrados específicos, la promoción de la investigación o la institucionalización de la igualdad a través del diseño y desarrollo de Planes de Igualdad (y Unidades de Igualdad) en las distintas universidades.

\footnotetext{
${ }^{1}$ En los últimos años son numerosos los artículos de opinión publicados en distintos diarios de tirada nacional que critican de forma contundente el giro neoliberal, mercantilista y devaluador del pensamiento libre y creador al que se debe la universidad. A modo de ejemplo, el publicado en eldiario.es el pasado 19/02/2016 titulado Disciplinar la investigación, devaluar la docencia: cuando la Universidad se vuelve empresa; o el publicado el 04/07/2014 por Marina Garcés en El País.es titulado ¿Están los estudiantes bien preparados? Compartimos los análisis y, en su mayoría, las opiniones expresadas en dichos artículos pero cuando nosotras utilizamos el término "crisis" no lo hacemos en ese mismo sentido, siendo sabedoras y protagonistas de dichos efectos, sino que más bien lo hacemos para poner el acento en el sentido estricto de cambio, transformación, paso de una situación a otra y, por tanto, en la oportunidad de creación.
} 
No obstante, la desigualdad en el contexto universitario sigue estando presente $^{2}$ y son múltiples los estudios que evidencian la debilidad de la presencia de materias que incluyan las relaciones entre hombres y mujeres desde diferentes disciplinas y ópticas históricas, culturales, lingüísticas... (Anguita y Torrego, 2008; Ventura, 2009; Vizcarra, Nuño, Lasarte, Aristizabal y Álvarez-Uria, 2015). En el caso de estudio que presentamos en este artículo, revisamos la posibilidad desde un enfoque metodológico concreto que los nuevos Grados de Educación Infantil y Primaria en la Universidad de Córdoba nos brindan para el desarrollo de los contenidos relacionados con coeducación, ética del cuidado e igualdad.

Un segundo factor, condicionante de posibilidad, para la docencia sobre igualdad y coeducación, lo situamos en el acento que el nuevo contexto universitario pone en los procesos de innovación. Estamos asistiendo a un impulso de nuevas propuestas docentes en la enseñanza superior (Rué, 2013; Ryan y Ryan, 2013) que coinciden en situar al y la estudiante en el eje central del aprendizaje, pasando el profesorado a ser quien organiza el proceso educativo en relación a aspectos cognitivos y sociales. Un ejemplo ${ }^{3}$ del modo como la innovación pasa a nutrir las prácticas docentes universitarias son los actuales Planes de Mejora Docente (o de Innovación Docente) promovidos desde la primera década del reciente siglo por las distintas universidades y cuyos beneficiarios y beneficiarias son el cuerpo de docentes. Estos programas vienen a diseñar e impulsar una línea de trabajo que orienta al profesorado universitario hacia la renovación de sus estrategias metodológicas y materiales de enseñanza.

El cambio en la concepción del trabajo del profesorado y del alumnado, enmarcado en la innovación, presupone una oportunidad, no solo porque idea una renovación en el ámbito de los recursos, sino porque reta la enseñanza

\footnotetext{
2 Sirva de ejemplo los diagnósticos de Igualdad elaborados por las distintas universidades del contexto español. A modo de ejemplo referenciamos del contexto andaluz el de nuestra Universidad de Córdoba disponible en

http://www.uco.es/igualdad/diagnostico/documentos/informe-creencias-igualdad-

alumnado.pdf; el de la Universidad de Málaga http://www.uma.es/media/files/263.pdf y la Universidad de Granada

http://unidadigualdad.ugr.es/pages/documentos/ diagnosticodegenerougr2011/!. Todos coinciden en señalar la existencia de un sesgo de género en la Universidad, afectando de forma desigual a las mujeres frente a los varones.

3 Otros ejemplos son la fructífera celebración de Jornadas, Congresos y encuentros relacionados con la innovación y la mejora docente universitaria que cada año se celebran en las Universidades públicas y privadas del país; o, también, la importante oferta de formación novel y permanente ofrecida por éstas en materia de metodología docente.
} 
tradicional y afecta a la propia cultura profesional incidiendo en las formas en las que se construye el conocimiento (López, Hinojosa y Sánchez, 2014). Por ello, tal como concebimos los procesos de cambio y mejora desde nuestro rol de docentes (García-Cano, Gutiérrez e Hinojosa, 2016), la actuación docente es una oportunidad para rescatar los intereses desde distintos posicionamientos del alumnado, partir de la (de $s u$ ) diversidad y pluralidad existente en el aula, más allá de entenderlo como un colectivo homogéneo. Y ello es coherente con un posicionamiento feminista y una formación para la coeducación ya que ideamos herramientas metodológicas de mejora que permitan al alumnado leer el mundo identificando la opresión y las desigualdades y, en tanto que sujetos políticos, también sean capaces de crear estrategias pedagógicas ligadas a los compromisos sociales y cívicos (Aguilar, 2013).

Estos factores son los que nos han servido de sostén para nuestra docencia con un firme posicionamiento en el reconocimiento de la desigualdad de género, las posibilidades formativas de la ética del cuidado, la coeducación y la igualdad entre hombres y mujeres en la práctica docente universitaria. Nuestro propósito en este artículo es reflexionar sobre la experiencia docente que venimos desarrollando durante dos cursos académicos (2014-15 y 2015-16) en el marco de la convocatoria de proyectos subvencionados por la Universidad de Córdoba en su III y IV Plan de Innovación y Mejora Educativa ${ }^{4}$. Describimos sus fundamentos teóricos y analizamos los aportes que su desarrollo tiene para la construcción de conocimientos coeducativos y sobre coeducación en el aula universitaria desde un enfoque reflexivo.

\section{FUNDAMENTOS TEÓRICOS: FORMACIÓN INICIAL DOCENTE, COEDUCACIÓN Y ÉTICA DEL CUIDADO}

Buena parte de los debates sobre educación e igualdad o coeducación, de forma particular para el campo escolar y la formación inicial del profesorado, surgen en relación a la dicotomía entre sexo/género, naturaleza/cultura y los modos en los que socialmente se estructuran los comportamientos e identidades "propias" de la masculinidad o feminidad en cada una de las sociedades. La identificación de estas diferencias se ha enmarcado desde la teoría feminista dentro de un orden social patriarcal, de poder y jerarquía, en el que ser mujer -atravesada por su posición de clase, etnia, religión,

4 Los proyectos subvencionados en ambas convocatorias han sido De la transmisión de contenidos a la arquitectura de experiencias. Dando contexto y significado al aprendizaje del aula en el espacio urbano (I) (ref. 2014-12-6013) durante el curso 2014-15 y II para el curso 201516 (ref. 2015-2-6006). 
(dis)capacidad, etc.- implica una subordinación y/o invisibilidad de ellas frente a los varones (Subirats, 1998). Los mecanismos pueden ser explícitos o muy sutiles pero en todos los casos las consecuencias implican el establecimiento de asimetrías y desigualdades de las mujeres con respecto a los varones (Lagarde, 1998).

La escuela, junto a la familia y los iguales, ha sido identificada como agente socializador determinante en la conformación de la identidad y categorización de género (Moreno, 2007). De ahí que numerosas investigaciones se hayan centrado en el estudio de cómo la escuela y el profesorado, a través de distintos mecanismos organizativos, el lenguaje, actitudes y concepciones reflejadas en el currículum oculto o los libros de texto configuran y contribuyen al mantenimiento de la estructuración de las diferencias y desigualdades generizadas y sexualizadas (Subirats y Brullet, 1990).

A pesar de la relevancia del profesorado en la construcción de las identidades de género, diferentes voces denuncian la parcial y escasa atención prestada a esta temática desde la práctica de la formación inicial docente (García et al., 2013; Bejarano y Mateos, 2014). Así lo describen Romero y Abril (2008) en el análisis que realizan sobre las metodologías y estrategias que se utilizan en la formación del profesorado de Educación Infantil para trabajar temas de género, dónde muestran que en el conjunto de centros analizados (a nivel estatal) las competencias de género no son destacadas como competencias profesionales importantes, no son prioritarias ni en los planes de estudios ni para las personas que coordinan estas titulaciones. Como señala Engebretson (2016), es común encontrar un discurso entre el profesorado manifestando su interés por la inclusión de contenidos de género para la formación docente pero, finalmente, la presencia de contenidos y perspectivas queda más bien a voluntad de profesorado individual, por lo que los y las docentes universitarios se muestran "desinformados aunque interesados".

Fruto de este interés mostrado por algunos y algunas docentes y del amplio desarrollo de los estudios feministas, identificamos dos líneas fructíferas de investigación que se han venido desarrollando en el ámbito concreto de la formación inicial del profesorado y que sirven de mimbre conceptual para justificar nuestra propuesta docente: en primer lugar, estudios interesados en la identificación y descripción de los discursos, estereotipos e ideas preconcebidas de profesorado en formación de las distintas etapas educativas sobre las diferencias, identidades y categorizaciones de los géneros; y en segundo lugar, una línea de trabajo de carácter más pragmático orientada a promover y analizar los efectos resultantes del diseño y puesta en práctica de 
materiales, planes de estudio o contenidos dentro de asignaturas vinculadas a la igualdad y coeducación. Nos detenemos en señalar los resultados de algunos de estos trabajos.

En alusión a los trabajos orientados a estudiar los estereotipos, prejuicios e ideas preconcebidas sobre las diferencias entre géneros y su configuración social, las investigaciones al respecto indican la existencia de discursos que establecen un "estándar" binario y cosificado de "ser hombre" y "ser mujer". La normatividad heterosexual y el privilegio masculino han sido puestos de manifiesto en la investigación desarrollada por Engebretson (2016) mediante el análisis feminista posestructuralista del discurso de futuros y futuras docentes de Educación Secundaria. En sus hallazgos se evidencian imágenes que asocian "ser hombre" con una condición normalizada, relacionada principalmente con el ámbito público, y "ser mujer" con una condición difícil y vinculada a la esfera doméstica o a la lucha por compaginar ambos espacios. En la misma dirección se manifiestan las percepciones de futuros y futuras docentes analizadas por Aslan (2015), caracterizadas por metáforas claramente patriarcales que describen "ser mujer" con adjetivos como delgada, sensible o necesitada de atención, mientras que "ser hombre" es definido generalmente como rudo, duro o insensible. Asimismo, estas percepciones tienden a ser más positivas hacia el género considerado propio que respecto al "otro".

Dentro de esta línea de trabajo, destacan también otras investigaciones que exploran los discursos y percepciones de futuros $y$ futuras docentes vinculando el género y la propuesta teórica de la ética del cuidado. Este conjunto de indagaciones se han preguntado, entre otras cuestiones, por las concepciones que los y las estudiantes tienen acerca del cuidado (Goldstein y Lake, 2000) o si su posicionamiento moral manifestado en la deliberación de problemas sociales complejos se sitúa más próximo a la ética del cuidado o de la justicia (Kohlmeier y Saye, 2012). En coherencia con los hallazgos de los estudios previos, los resultados en este caso apuntan hacia estudiantes con razonamientos más próximos a la ética de la justicia, tanto en el caso de los varones como de las mujeres y una consideración esencialista, simplista e idealizada del cuidado, como un elemento más propio del instinto que del aprendizaje, relacionado con la maternidad y un ideal de docencia feminizado y afectuoso (Goldstein y Lake, 2012).

En España son muchos más los trabajos que se han publicado sobre el debate teórico y los principios del razonamiento moral, diferencias o complementariedad existente entre la propuesta Kohlberg y la ética de la 
justicia y la de Gilligan y la ética del cuidado (Fascioli, 2010; Gonzálvez y Jover, 2016; Vázquez y Escámez, 2016). Aún son escasas las investigaciones en el ámbito de la formación universitaria que exploren acerca de los discursos de estudiantes o profesorado al respecto o analicen propuestas docentes inspiradas en los principios de la ética del cuidado. Una excepción la encontramos en el trabajo dirigido por Torras (2013) desarrollado en la Universidad Oberta de Catalunya que analiza los discursos escritos (a partir de comunicaciones electrónicas y documentos) de estudiantes y profesorado en asignaturas desarrolladas on line mediante la aplicación de metodologías cooperativas. El trabajo destaca las potencialidades metodológicas al promoverse la interdependencia, comunicación, responsabilidad y cuidado cercanos a la ética del cuidado entre los y las participantes.

En segundo lugar, y sobre todo a partir de los procesos de transformación de los estudios universitarios con el EEES, encontramos un importante número de trabajos que describen y analizan los efectos de la incorporación de materias y contenidos relacionados con el género. En todos éstos se parte de la premisa de la ausencia e invisibilidad de esta temática en las universidades ya descrita.

Estas experiencias transitan desde actuaciones a nivel macro o de planes de estudios (las menos numerosas), como los Grados de Educación Primaria e Infantil en la Escuela Universitaria de Magisterio de Vitoria-Gasteiz (Vizcarra et al., 2015) o la formación docente en las Facultades de Educación de Ciudad Real y Toledo (Bejarano y Mateos, 2014), hasta innovaciones producidas en asignaturas concretas. Es especialmente en estas últimas dónde se ha centrado el interés por analizar el impacto que han generado en los y las futuras docentes. Los resultados, de manera general, apuntan hacia efectos positivos en cuanto al cambio de actitudes, una mayor concienciación o el incremento de conocimientos respecto al género y sus implicaciones en la docencia.

El estudio conducido por Case, Hensley y Anderson (2014) se sustenta en una propuesta formativa para aumentar la conciencia sobre los privilegios masculinos y heterosexuales y reducir los prejuicios del alumnado. La propuesta incluye documentos que evidencian los privilegios, videos testimoniales y escritura reflexiva. Con un diseño experimental en el que han participado 303 estudiantes, descubren que las estrategias implementadas, especialmente la audiovisual, aumentan la conciencia sobre los privilegios mencionados en el alumnado. También con resultados muy positivos, describen una propuesta formativa coeducativa Fernández y Piedra (2010) en el ámbito de la Educación Física. Su experiencia se estructura en dos partes: 
una destinada a sensibilización y otra a la aplicación de la teoría Doing Gender, subrayando las manifestaciones de género relacionadas con la actividad física y el deporte. El pretest y el postest aplicados evidencian un efecto positivo de la formación, mostrado en un aumento de las puntuaciones medias y, con él, un cambio en las actitudes iniciales del alumnado participante. El trabajo pre-experimental de García et al. (2013), si bien señala efectos positivos de la innovación desarrollada (de carácter transversal) respecto al aumento de conocimientos y de los niveles de metacognición del alumnado, también alude a que los niveles alcanzados continúan siendo bajos. La mayoría del alumnado participante, aunque de manera más acusada en el grupo control, muestra necesidades muy básicas en su formación sobre género y homofobia, tanto en conocimientos como en la falta de coherencia y realismo en sus posturas.

Los resultados de estos trabajos manifiestan con rotundidad la necesidad de incorporar cuestiones de género en la formación inicial docente, al tiempo que alientan sobre las posibilidades de la formación y los efectos positivos que puede producir en el futuro profesorado. Nuestro posicionamiento acerca de cómo innovar desde la perspectiva de género, como docentes feministas en los estudios de formación de maestros y maestras, parte de una consideración identificada por Butler $(2001,8)$, central para la teoría feminista: el pensamiento sobre la vida y su relación con la transformación social. Cuando hablamos de la vida, nosotras, nos referimos a pensar la convivencia, las formas de relación y organización de posiciones y roles. Y tal como la teoría feminista posestructuralista acentúa, poniendo en cuestión la relación entre diferencia sexual y categorías de mujer y hombre o del propio concepto de género como concepto unívoco y esencialista. Como señala la autora:

no existe un mero enfoque epistemológico hacia el género, una manera simple de preguntar cuáles son las formas de conocer de las mujeres o qué podría significar conocer a las mujeres. Por el contrario, las formas en que se dice que las mujeres "conocen" o "son conocidas" están ya orquestadas por el poder, precisamente desde el momento en que los términos de categorización "aceptables" son instituidos (Butler, 2001, 16).

Por ello, nos servimos de la teoría feminista y el concepto de género como un mecanismo para pensar y hacer pensar la convivencia (la vida) dentro de nociones que naturalizan, normativizan o regulan lo hegemónico. Coincidimos con la teoría feminista en la necesidad de dotar a los maestros y maestras en formación de estrategias intelectuales que les permitan resignificar los conceptos de convivencia, igualdad, cultura de paz o cuidado desde distintos lugares y contextos, desde distintas posiciones y experiencias. 
La propuesta de enseñanza desde la "ética del cuidado" inspirada en la teoría moral de Gilligan (1994) supone una apuesta en este sentido de cambio y resignificación, el establecimiento de relaciones significativas y el compromiso de responder a las necesidades del "otro" con responsabilidad, sensibilidad y flexibilidad. En sí misma conduce a un reordenamiento de las prioridades escolares, desde la prevalencia de la capacitación académica exclusivamente, hasta una educación que atienda a las personas tanto en su capacidad de cuidar como en su manifestación de seres que necesitan ser cuidados (Noddings, 2009, 40), lo que en nuestra práctica docente significa optar por metodologías que transcienden el mero acto intelectual, pasando a ser una elección moral por parte de nuestro alumnado y de nosotras mismas como docentes. En la filosofía moral encontramos dos concepciones diferentes de la otredad que fueron denominadas por Seyla Benhabib como "el otro generalizado" y el "otro concreto" (Fascioli, 2010; Fernández y López, 2010). La ética de la justicia pone el acento en la imparcialidad, la universalidad vinculada a la perspectiva de un "otro" generalizado, abstraído de su identidad, mientras que la ética del cuidado enfatiza el punto de vista del "otro" concreto, subjetivo, con rostro y cuerpo. Desde la primera postura, las relaciones con el "otro" parten de concepciones imaginadas, estereotipadas y universalizadas, desconociendo su contexto específico; sin embargo, desde la ética del cuidado no sólo se establece una relación dialógica sino una relación de atención y cuidado que nace y se nutre de las necesidades reales que presentan las personas concretas.

Conocer, acercarse y relacionarse con este "otro concreto" abre la puerta a la generalización del cuidado como un verdadero valor humano y no una cuestión de género (femenino) y posibilita la ruptura de visiones alienadas y alienantes del género, así como la construcción de roles más flexibles, aspectos ambos esenciales para Comins (2003) a la hora de conformar nuevas formas de interacción más equitativas y alternativas viables a las actitudes y comportamientos sexistas, violentos y patriarcales.

El cuidado, desde este prisma, trasciende el ámbito privado dónde tradicionalmente ha sido ubicado para convertirse en un proceso social, público. Aprender a pensar en los demás concretos desde nuevos marcos de relación basados en el cuidado, puede funcionar, así, como motor de un cambio social más amplio, al verse implicados e implicadas, comprometidas con un "otro" que no es lejano, sino interdependiente a la existencia y vivencia propias. Ese sentimiento de unidad global y responsabilidad hacia los demás puede generar una mayor participación social, redefinida desde una ciudadanía más equitativa, inclusiva y menos androcéntrica (Comins, 2008). 
Introducir estas nuevas formas de relación, comprensión y construcción del género en la formación inicial docente requiere indudables transformaciones que han sido clasificadas por García et al. (2011) en tres niveles: sociocultural, interactivo e individual. El nivel sociocultural involucra cambios en las políticas universitarias, en la organización institucional, el reparto de responsabilidades y los planes de estudios. El nivel relacional, más cercano a la práctica de aula, incorporaría transformaciones en las relaciones que se establecen entre personas y con el conocimiento (en su construcción y significado), el uso del lenguaje, las propuestas curriculares y las prácticas concretas que se ofrecen al alumnado. Por último, a nivel personal, conlleva un replanteamiento de las propias creencias y expectativas docentes. Como se aprecia, no se trata de transformaciones que puedan ser solventadas con la introducción de una asignatura particular, un contenido específico o una práctica puntual, sino que requieren de un proceso innovador más complejo, sistémico y profundo, asuntos ya adelantados entre otras por DonosoVázquez y Velasco-Martínez (2013), Freixas, Fuentes-Guerra y Luque (2007) o Aguilar (2013), en relación a la formación inicial del profesorado.

Describimos de forma detallada nuestra propuesta de diseño docente para a continuación analizar las oportunidades que nos brinda en la construcción de aprendizajes coeducativos y sobre coeducación.

\section{NUESTRA EXPERIENCIA DE INNOVACIÓN DOCENTE: CONSTRUYENDO APRENDIZAJES DESDE EL AULA Y EL ESPACIO URBANO}

Nuestro proyecto de innovación docente se ha desarrollado en la asignatura "Convivencia Escolar y Cultura de Paz", impartida en segundo curso de los Grados de Educación Infantil y Educación Primaria de la Universidad de Córdoba. En los dos cursos en los que se ha implementado el proyecto (durante el 2014/15 y 2015/16), se han involucrado todos los grupos en los que se impartía la materia (4 grupos de Educación Primaria y 3 de Educación Infantil). Se trata de una asignatura anual cuyos contenidos versan sobre cuestiones como: condicionantes sociales de la convivencia escolar; educación en valores y cultura de paz; diversidad e igualdad de oportunidades; o programas y estrategias para la convivencia en la escuela.

El origen de esta asignatura se sitúa en el marco institucional descrito, concretamente en el proceso de conformación de los nuevos Planes de estudio de Grado adaptados al EEES. A pesar de la insuficiente incorporación de 
materias que aborden contenidos relacionados con el género, la coeducación y la equidad entre géneros en los Grados de formación inicial del profesorado, como se ha mencionado con anterioridad, esta asignatura ha supuesto una oportunidad al integrar descriptores y contenidos en este sentido, haciéndolos más visibles y explícitos.

Esta oportunidad se tradujo, desde el inicio, en una serie de prácticas docentes que enfatizaban los aspectos relacionados con la diferencia sexual y la igualdad de oportunidades, como la visualización de películas, lectura de documentos, etc. Sin embargo, como ha ocurrido a menudo (Vizcarra et al., 2015), aunque útiles, estas prácticas seguían resultando anecdóticas, puntuales, no centrales en su formación y no integradas en una estrategia de conjunto. Daba la sensación de que se trataba de un contenido más, separado del resto del programa y separado, asimismo, de la realidad y la experiencia del propio alumnado como agente activo, social y políticamente, como ciudadano y ciudadana. El alumnado no sentía estos contenidos como algo vinculado al contexto social y cultural que estábamos analizando, del que ellos y ellas forman parte y desde el que se configuran sus experiencias, sino como una cuestión que afecta a otras personas en otros lugares.

Estas distancias, tanto epistemológicas como vivenciales, hicieron que las docentes que impartimos la asignatura sintiéramos la necesidad de mejorar nuestra práctica aplicando los presupuestos teóricos de la coeducación y la ética del cuidado, ofreciendo a nuestro alumnado oportunidades para comprender y desarrollar la ciudadanía desde esta perspectiva. De esta manera buscamos que la distancia emocional se tradujera en vivencia y la desconexión entre contenidos se transformara en una propuesta formativa cuyo eje central fuera el acercamiento a los "otros" y las "otras", viéndose interpelados e interpeladas por su realidad. En definitiva, relacionar la formación académica de aula y la proyección personal y social de los aprendizajes, con la responsabilidad y el sentimiento de interconexión con la comunidad tanto local como global (Comins, 2003).

Con este propósito, ideamos un proyecto de innovación docente con el objetivo de que nuestro alumnado comprendiera la escuela en el marco del contexto social más amplio, y asuma la importancia de incorporar a la comprensión de lo que ocurre en las aulas el análisis crítico de las cuestiones más relevantes de la sociedad actual, que afectan a la educación en general y a la escolarización en particular, y entre las que encuentra un lugar prioritario las relaciones entre géneros. Asimismo, concebíamos la docencia como una profesión transformativa (Giroux, 1990) que requiere de maestros y maestras 
activistas, comprometidas y críticas, por lo que entendíamos prioritaria su capacitación para percibir las desigualdades provocadas por la sociedad patriarcal y androcéntrica en la que vivimos y apostar de manera decidida por prácticas coeducativas en su futura práctica profesional (Rebollo, 2013).

Todo aprendizaje, más aún cuando se trata de aspectos no sólo cognitivos, sino también actitudinales y axiológicos, implica aspectos emocionales. No se trataba, por tanto, de consumir información (el contenido de la asignatura), contemplar o sentirse indiferente ante ésta, sino más bien que el alumnado se sintiera "afectado" por la realidad que aprende, poniendo cuerpo y cara a dicho contenido. Esta vinculación entre desafíos intelectuales y emociones resulta imprescindible para que los aprendizajes sean profundos, interconectados y se mantengan en el tiempo (Cochran-Smith, 2009).

Con todos estos propósitos como punto de partida, el proyecto de innovación docente ha incorporado una serie de actuaciones que el alumnado, en grupos, ha ido desarrollando a lo largo de la asignatura:

1. Ha establecido un proceso de relación con organizaciones, asociaciones y colectivos del ámbito social y cultural en la ciudad que le ha permitido construir conocimientos colaborativamente y significar los contenidos abordados en la asignatura. Ha identificado dichos colectivos, contactado y negociado la entrada al campo de estudio con ellos.

2. Ha indagado a través herramientas de investigación social (entrevistas, observaciones y análisis documental) en el trabajo desarrollado por los colectivos para conocer en primera persona su realidad (objetivos, demandas, problemáticas, estructura de organización interna, etc.).

3. Ha elaborado un ensayo en el que ha establecido vínculos conceptuales y reflexivos entre los hallazgos de las indagaciones de campo y los contenidos de la asignatura.

4. A la luz de las necesidades detectadas en el colectivo analizado, ha realizado un recurso didáctico para devolver a la organización o bien para dar a conocer el trabajo realizado por dicho colectivo en el ámbito de la educación Infantil o Primaria.

5. Ha compartido su trabajo (tanto la indagación como el recurso elaborado) con compañeros y compañeras, colectivos implicados y comunidad educativa 
en general en una feria de "stands" que tuvo lugar en espacios abiertos de la Facultad de Ciencias de la Educación (hall, pasillos, etc.).

Paralelamente, estas actuaciones del alumnado se han articulado con un proceso docente compuesto por las siguientes fases:

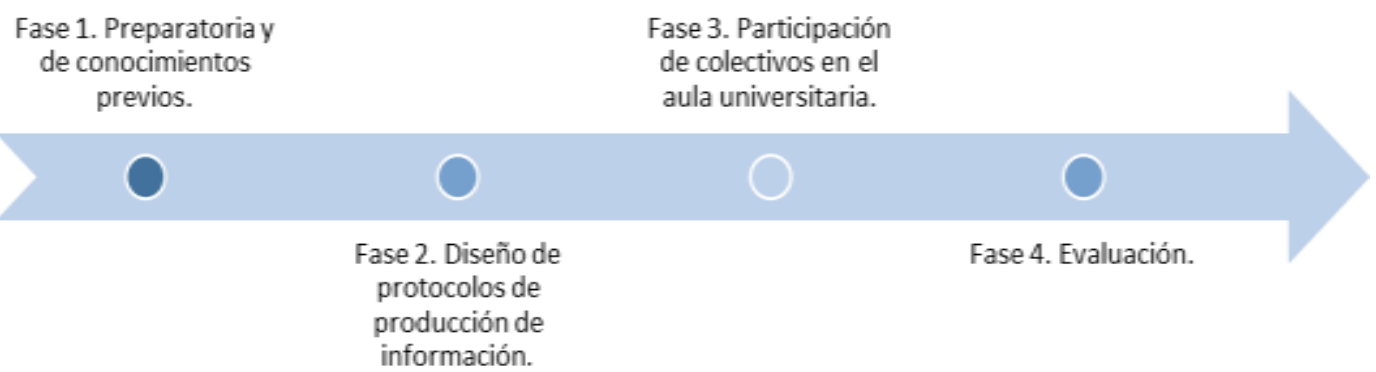

Figural. Secuencia de fases del proyecto de innovación docente

Fase 1. Preparatoria y de conocimientos previos. Las docentes hemos indagado en las experiencias previas del alumnado con colectivos y organizaciones sociales de la ciudad a través de la aplicación de un cuestionario y la realización de grupos focales. Además, en estos momentos iniciales se presentaron al alumnado las tareas a realizar, los objetivos pretendidos y se dedicaron diferentes seminarios para explicar la metodología a seguir.

Fase 2. Diseño de protocolos de producción de información. En dicha fase el contenido teórico de la asignatura se desarrolló en paralelo al trabajo de campo realizado por el alumnado. Para las indagaciones, el alumnado fue acompañado por las docentes en la elaboración de protocolos para la producción de información a partir de tres estrategias de investigación social: las entrevistas, la observación y el análisis documental. En todo momento se ha procurado que la acción docente se desarrolle bajo una metodología centrada en el alumnado, protagonista de su aprendizaje, y en su participación activa.

Fase 3. Participación de colectivos en el aula universitaria. De forma intermitente se ha contado con la participación de algunos de los distintos colectivos con los que el alumnado ha contactado, con el objeto de motivar y promover la curiosidad compartida. En los dos cursos de desarrollo de la propuesta han sido numerosas las organizaciones participantes, entre las que cabe destacar, por su compromiso en la lucha contra las desigualdades de 
género, "Mujeres en Zona de Conflicto", "Paz con Dignidad", "Ingeniería Sin Fronteras", "Plataforma Cordobesa contra la violencia de las mujeres", "Acampada por la Dignidad. Centro social Rey Heredia".

Fase 4. Evaluación. La evaluación de la acción docente y del alumnado ha sido una tarea colectiva en la que ha participado tanto el alumnado como el profesorado mediante la elaboración de rúbricas completadas de forma compartida. Además, se ha vuelto a aplicar el cuestionario y la realización de grupos focales con el objeto de evaluar el proyecto de innovación y los aprendizajes producidos.

Esta práctica docente implementada durante dos cursos académicos ha servido de escenario privilegiado para el análisis que a continuación presentamos.

\section{APORTES DE LA EXPERIENCIA DOCENTE PARA LA CONSTRUCCIÓN DE CONOCIMIENTOS COEDUCATIVOS Y SOBRE COEDUCACIÓN EN EL AULA UNIVERSITARIA}

Diferentes han sido los aportes que esta metodología docente nos ha proporcionado en el ámbito de la coeducación en la formación inicial de maestros y maestras de Educación Infantil y Educación Primaria. A partir de un procedimiento de reflexión sobre nuestros postulados de partida, pero también de la práctica docente y de las distintas reconstrucciones que hemos ido creando, señalamos los que nos parecen más relevantes siguiendo el esquema analítico aplicado por Torras (2013) y adoptado de Gilligan (1982) en relación a la enseñanza de la ética del cuidado y que sistematiza en los siguientes elementos: interdependencia, comunicación, responsabilidad y cuidado.

Interdependencia. El punto de partida de nuestro planteamiento ha sido proporcionar aprendizajes holísticos, integrados, que superen enfoques puntuales sobre igualdad y diferencia sexual. A pesar de ser desarrollado en una sola asignatura, el enfoque propuesto incorpora herramientas para el análisis de la escuela desde lo global. Para ello, partimos de la concepción feminista del conocimiento situado, que necesita entrar en diálogo y atender a quienes sufren opresión con el objeto de pensar en modelos de sociedad y en modelos de escuela. Este pensamiento reflexivo, más allá de hacerse desde lo abstracto, alejado de la cotidianidad y aparentemente separado de la experiencia vital del estudiantado, es inspirado desde la implicación que 
supone ponerse en contacto con colectivos sociales que se definen en consonancia con objetivos y demandas sociales y políticas vinculadas a su opresión o desigualdad. El ejercicio que hacemos provoca un cambio de localización del alumnado, promoviendo la sensibilidad ética y política y situándolo en un nuevo posicionamiento que trasciende, desde la mera expectación, al contacto y estudio de problemáticas sociales desde dentro del propio colectivo.

Este sentido, la interdependencia también se refleja en la labor docente. Y así lo manifestamos el grupo de profesoras que impartimos la asignatura en nuestros diarios de clase $^{5}$ elaborados durante el curso. Una profesora reflexionaba sobre su rol de docente y la necesaria interacción e interdependencia que establecía con los colectivos sociales, y cómo su labor le exige una actuación y compromiso más allá del aula universitaria.

¿Qué les pido a los colectivos sociales y qué puedo hacer desde mi posición de profesora universitaria? Entiendo que mi posición puede percibirse por su parte como un tanto privilegiada, tanto por la visión de prestigio de la ocupación [docente universitaria] como por el "poder" de llegar a un colectivo muy amplio [amplio número de estudiantes, jóvenes futuras docentes], pero sobre todo porque los conocimientos que yo diseñe y transmita están legitimados por el propio escenario y estructura institucional desde la que los transmito (la Universidad). En este sentido debo tener muy presente que yo también me estoy implicando con los colectivos sociales, paso a formar parte del entramado de interacción que le solicito en la tarea académica a mi alumnado y de alguna forma he de cuestionarme/reflexionar/actuar ante los aprendizajes que estamos construyendo (dentro-fuera) (D1516MG).

El sentido relacional, dialógico y cercano es compatible con la propuesta de Benhabib (2011) acorde a pensar el otro como "el otro concreto", con rostro, presente en sus demandas y estrategias.

El panorama de colectivos con los que el alumnado ha trabajado atraviesa el ámbito de la salud, la exclusión social y grupos marginados, discapacidad y dependencia, defensa de los Derechos Humanos, de los animales, cooperación,

5 Hemos desplegado distintos instrumentos de investigación durante estos dos cursos académicos de implementación de la innovación docente: cuestionarios ad hoc, grupos focales al inicio y final de curso con los y las estudiantes y también entre las profesoras y realización de diarios de clase por nuestra parte. No es nuestro objetivo aquí presentar un análisis sistemático de dicha información ya abordada en otros trabajos en prensa. En este artículo rescatamos alguna de la información producida en nuestros diarios con el objeto de ilustrar y apoyar nuestras reflexiones sobre la intervención docente. 
ocio y cultura y la educación. En todos los casos promovemos un tipo de análisis social y educativo a la luz de los condicionantes sociales actuales, entendiendo que las motivaciones, demandas y dificultades de los colectivos sociales quedan constreñidas por factores del contexto histórico y político. Este acercamiento permite que el alumnado comprenda que ciertas demandas particulares deben ser abordadas desde el reconocimiento social y que la propia escuela juega un papel fundamental en él con decisiones tomadas al planificar el curriculum, incorporar ciertos contenidos, silenciar otros, problematizarlos o presentarlos de forma aparentemente neutra.

Así, el proceso de enseñanza-aprendizaje desde una perspectiva coeducativa permite pensar las problemáticas sociales más allá de la mera identificación de hombres y mujeres en el escenario educativo o entendiéndolo exclusivamente como una cuestión lingüística o de (in)visibilidad, sin lugar a dudas necesario y de máxima relevancia. Este enfoque creemos que complejiza el enfoque coeducativo pues no queda supeditado a las dicotomías sexo-biológico /género-cultura para pasar a entender el cuidado, como insiste Comins (2008), como un asunto público. Así, ha sido de interés observar cómo el alumnado ha permanecido atento a las posiciones de poder entre personas y grupos, a las jerarquías generadas en las relaciones cotidianas, visibilizando tareas y saberes populares. Pero sobre todo, el alumnado ha observado a personas en situaciones de vulnerabilidad y ha reflexionado sobre la importancia de comprenderlas en el marco de complejas relaciones contextuales e históricas. También ha advertido cómo grupos concretos ejercen la ciudadanía desde una posición de interdependencia, acción y cuidado a las personas, creando sociedad, culturas y ciudadanía que de otra forma raramente se nos muestran.

Comunicación. El diálogo se ha establecido como fuente de relación y eje en torno al cual se han articulado los aprendizajes. Éste no solo se ha establecido dentro del aula, a través de la realización de trabajos grupales que obligan a la discusión y debate intra grupo, sino además, por la propia metodología que se ha establecido para el desarrollo de la tarea académica con los colectivos sociales. El alumnado ha experimentado el proceso de negociación en el acceso al campo y también para su conocimiento e indagación. Este diálogo se ha pretendido de forma horizontal, no experta, basado en el aprendizaje. Noddings $(2009,45)$ lo define como uno de los componentes fundamentales de la educación moral, necesaria para el desarrollo de la ética del cuidado. Una comunicación basada en la escucha, en la que el sujeto esté dispuesto a cambiar su punto de vista, su posicionamiento de partida. 
Nuestra experiencia docente implica al alumnado en la realización de varias entrevistas a distintos actores de la organización seleccionada, de forma que le permita contrastar puntos de vista, pero además, conocer las distintas experiencias con respecto a la organización de la que forman parte. El objetivo de ese contraste ha servido para fundamentar interpretaciones y conocer distintas posiciones desde la que cada uno y cada una habla y verbaliza su experiencia. Esta oportunidad de comunicación y diálogo compartido ha abierto el interés en el alumnado por la vida ajena, por sacar a luz experiencias cotidianas, personales, de sujetos particulares. De esta forma, no solo hemos trabajado el contenido relacionado con la coeducación y la igualdad, sino que la metodología nos ha permitido poner en valor lo relacional y también la emotividad. Tal como proyectan en su trabajo Freixa, Fuentes-Guerra y Luque (2007) el conocimiento de situaciones desconocidas, ajenas, que transcienden lo racional y generan choque y proximidad es una estrategia metodológica de alto valor para el desarrollo de la coeducación en la formación inicial del profesorado. Y este efecto sobre todo se ha producido en la descripción e interpretación de grupos y asociaciones que se organizan con el objeto de dotar bienestar físico y emocional a las personas, como ha sido el caso con el análisis de organizaciones de personas con discapacidad, voluntariado que trabaja en asociaciones de afectados por el cáncer o colectivos de mujeres contra la violencia de género. En uno de los diarios de las profesoras se exponía de la siguiente manera:

En las primeras clases me doy cuenta que el alumnado también está ilusionado con lo que vamos a hacer, así lo comentan también en los grupos focales iniciales que hemos realizado. Les gusta aprender fuera de las aulas, les gusta conectar sus aprendizajes teóricos con lo que sucede en la sociedad. Hay pocas personas que hayan colaborado con asociaciones anteriormente pero exponen sus experiencias y el resto de alumnado atiende, me da la impresión que tienen necesidad de aprender de lo que les cuentan sus compañeros (D1516EG)

Responsabilidad. En el desarrollo de la asignatura el alumnado ha transitado desde posiciones que argumentan la falta de participación, compromiso y responsabilidad social con la ausencia de tiempo o interés, hasta posturas que ponen en cuestión estos argumentos haciendo patente cierto sentimiento de unidad, de preocupación e implicación con las situaciones ajenas de desigualdad. Lo que le ocurre a los y las demás comienza a considerarse como algo relacionado, en buena medida, con nuestra propia acción. Así, las motivaciones por generar cambios sociales (y educativos) se sustentan en un plano más intrínseco que extrínseco, más concreto que regido exclusivamente por principios generales que se nos pueden plantear lejanos y abstractos. Esta es una cuestión fundamental en una enseñanza coeducativa coherente con la 
ética del cuidado, ética que Comins (2008) acertadamente denomina "de la responsabilidad", al tener en su base el principio de equidad y el reconocimiento de las necesidades de los demás, que da lugar al cuidado, y que complementa otros postulados éticos más igualitaristas que modulan y adaptan las demandas específicas de los otros y de uno mismo a principios universales. Plantea la autora:

La ética de la justicia nos recuerda la obligación moral de no actuar injustamente con los otros, la ética del cuidado nos recuerda la obligación moral de no abandonar, de no girar la cabeza ante las necesidades de los demás (Comins, 2008, 15).

Sentirse parte del cambio desde lo personal, desde nuestra forma de ser y estar en el mundo, contribuye también a forjar la idea de que todas las personas en todos los momentos de nuestras vidas somos cuidadores al tiempo que demandantes de cuidados, porque nuestras vidas son $\mathrm{y}$ pueden ser vulnerables. Este giro por parte del alumnado fue percibido por el profesorado que lo reflejaba en su diario de clase:

Durante el desarrollo de mi reflexión una alumna ha levantado la mano y ha expuesto en algo su experiencia personal con respecto al trabajo de campo que desarrollan en Convivencia. Ha explicado cómo su contacto con la asociación y las distintas visitas que están realizando al barrio (un contexto de alta marginalidad en la ciudad) le está permitiendo cambiar su percepción sobre el contexto. Ha narrado como en sus primeras visitas todo el grupo sentía miedo al acercase, dejaban el coche lejos para evitar que les "ocurriera algo", temían por ellas y realmente no sabían muy bien qué podrían aprender de dicha situación. A medida que han visitado el barrio y han conocido la intervención pedagógica sus miedos se han disipado, descubriendo cómo ellas también tenían mucho que aprender tanto de la organización como de la gente con la que trabajan. Ya no tenemos miedo en acudir, señala. "Eso no es lo que nos parece más importante. El miedo a lo desconocido, nos imaginábamos lo peor de aquel barrio y a medida que hemos conocido a la gente estamos mucho más relajadas y conscientes de que no hay tantas diferencias con lo que ocurre en otros lugares. Nuestros miedos se debían más a lo desconocido y no lo veíamos como un contexto donde podían pasar experiencias para aprender" (D1516MG)

Estas transformaciones atraviesan los niveles de cambio relacionales y personales señalados por García et al. (2011) como imprescindibles en una formación inicial del profesorado coeducativa, al motivar otras formas de relación más comprometidas y solidarias, así como al evidenciar un cambio en las posiciones de partida. Cambio del que las docentes no somos ajenas, pues 
impregna nuestra labor diaria y nos interpela también a nosotras, haciéndonos reflexionar sobre nuestros posicionamientos de partida, sobre cómo nos mostramos al alumnado y nuestras actitudes como cuidadoras y demandantes de cuidado. En definitiva, sobre nuestra propia responsabilidad respecto de la formación que promovemos en las aulas. Así lo manifestaba una de las docentes implicadas en la experiencia de innovación a través de las reflexiones vertidas en su diario de campo:

Hace poco tuve una discusión con un familiar sobre la formación de actitudes y valores en la educación formal. Comenzó por la educación en primaria y terminó con la universidad. Veo que sigue manteniéndose, incluso en maestros en ejercicio, la idea de que podemos (y debemos) ser "neutrales". Que lo contrario es adoctrinar y que quiénes somos nosotros/as para poder decidir sobre los valores que transmitimos o que son mejores para construir una sociedad futura. Esta discusión me hizo reflexionar mucho sobre la asignatura y sobre todo, sobre su necesidad. Nadie puede ser neutral y sí hay una serie de valores que contribuyen en mejor medida a la convivencia pacífica. Creo que esto debe quedar patente en la formación inicial del profesorado. Tener claro hacia dónde vamos, qué modelo de ciudadano y ciudadana, de persona, queremos contribuir a construir, desde qué perspectiva axiológica e ideológica. Es un debate necesario y una reflexión ineludible. No podemos seguir excusándonos en que "debemos ser neutrales". Mientras no "nos mojamos", se siguen reproduciendo una y otra vez patrones, estereotipos, contravalores, odios y venganzas que poco ayudan a la educación, al desarrollo de una persona en todo su ser y en sociedad (D1516EH).

La responsabilidad, desde este prisma, nos lleva a reflexionar sobre nuestra actuación docente y sobre nuestro pensamiento, haciéndonos conscientes de la importancia que adquieren en un proceso formativo que busca construirse desde parámetros coeducativos de una manera deliberada y explícita.

Cuidado. La breve pero intensa experiencia desarrollada por el alumnado a lo largo de la asignatura le ha invitado a desarrollar acciones de cuidado de una manera activa, práctica, real. Este elemento ha sido muy evidente en el trabajo de aula con el alumnado, especialmente en la tarea relativa a la elaboración de un recurso didáctico vinculado a las necesidades detectadas, de manera que supusiera una devolución a los colectivos con los que han trabajado. El alumnado ha partido de sus recursos, de su tiempo, de sus conocimientos y se ha puesto en disposición de dar. Así por ejemplo, los grupos que han trabajado con "Mujeres en Zona de Conflicto" han creado un cuento para sensibilizar y trabajar en los centros educativos en contra de la violencia contra las mujeres, y el grupo que ha trabajado con "Acampada por 
la Dignidad. Centro social Rey Heredia" ha realizado un video promocional de sus objetivos y actuaciones. Con sus recursos y conocimientos todos los grupos crean su forma concreta de devolución para que no quede como un material solo para aprobar la asignatura, sino útil y utilizable por los colectivos con los que han interaccionado.

Otro cauce en el que se ha visto reflejada la disposición de cuidar en el alumnado ha sido en la exposición final de los trabajos realizados, lo que ha funcionado como mecanismo de difusión de las distintas entidades y de sus acciones, visiones y aportes. Esta exposición, desarrollada a modo de feria de stand en el hall de la Facultad, supuso un acto transparente y abierto de compartir el conocimiento construido con toda la comunidad universitaria y con los colectivos sociales. Así lo señala una de las docentes en el grupo focal realizado por el profesorado al término del proceso de innovación, donde describe el impacto que ha tenido este acto de compartir y difundir en el seno de la comunidad universitaria:

En el proyecto fíjate que al final todo tiene su trascendencia. Una alumna de máster, que va a hacer el trabajo fin de máster conmigo, me dijo que va a hacer sus prácticas de máster en una asociación porque la vio en la feria de asociaciones (...) Me dijo "la vi, y de todas las que vi me gustó esa y he ido para interesarme y hacer mis prácticas de TFM allî" (GF1516EH).

Para responder a ambas acciones de devolución (elaboración del recurso y difusión de trabajos), ha sido imprescindible para el alumnado preguntarse qué puede hacer como estudiante y como futuro y futura docente para dar respuesta a las necesidades que presenta el colectivo con el que han trabajado. Cuestionamiento y acción que quedan vinculadas al componente de la educación moral que desde la ética del cuidado Noddings (2009) denomina como "práctica" y se concreta en la capacidad de atención interpersonal, en la capacidad de cuidar. Para ello resulta necesario el establecimiento de relaciones con las personas que necesitan cuidado, el conocimiento de sus demandas y la adecuación de nuestra acción a ellas. Aunque tradicionalmente asignada a las mujeres, la práctica del cuidado desde la perspectiva coeducativa es propia de todas las personas independientemente de su género y del espacio en el que se desarrolle. Así lo hemos entendido y fomentado desde nuestra propuesta docente, al promover una práctica del cuidado en todo nuestro alumnado y en diferentes espacios de encuentro (en el aula universitaria, en las sedes de los colectivos o en sus áreas de actuación, etc.). Esta postura supera la asignación patriarcal de roles de género y favorece una 
reconstrucción de los mismos más flexible, menos estereotipada, menos asimétrica.

\section{A MODO DE CONCLUSIÓN}

A lo largo del artículo se ha reflexionado acerca de los aportes y el potencial coeducativo que desde la ética del cuidado presenta un proceso de innovación docente desarrollado en la Universidad de Córdoba, en los Grados de Educación Infantil y Primaria. Se contextualiza en el momento actual de consolidación de los nuevos estudios de Grado y las posibilidades que ofrece el marco normativo universitario para la incorporación de contenidos y asignaturas en las que se aborden temas de género y coeducación. Se advierte, no obstante, la escasa presencia que adquieren estas cuestiones en la práctica real de la formación inicial docente, a pesar de los esfuerzos e impulsos generados desde el desarrollo legislativo, la investigación y la teoría feminista.

Convertirse en docente, aprender a enseñar, comporta indudablemente adquirir conocimientos de diversa índole, pero estos conocimientos no son asépticos, sino que se enmarcan en estructuras sociales, políticas y económicas más amplias y están impregnados de valores, actitudes, emociones, etc. Cómo se construyan y se transmitan juega un importante papel, pudiendo convertirse en una fuente de transformación social y educativa o en un mecanismo de reproducción de desigualdades y opresiones.

La innovación descrita se ha desarrollado bajo fundamentos que enfatizan una construcción de conocimientos desde una perspectiva más próxima y horizontal, a través de la intersección de distintas voces y demandas, y donde se han incorporado elementos como la interdependencia, la comunicación, la responsabilidad y el cuidado. Tanto los aportes generales del feminismo al campo de la formación inicial del profesorado, como concretamente las contribuciones del modelo educativo de la ética del cuidado cobran un papel protagonista y abren la puerta a una formación coeducativa, no sólo en el plano conceptual, sino también en otros ámbitos más tácitos que a menudo quedan ocultos y se concretan en la transformación de las relaciones que mantenemos con los demás, en nuestra moral, acciones de cuidado y posicionamientos. Queda pendiente convertir estas acciones educativas en el núcleo central de los planes de formación de maestros y maestras para que sean también el eje de la educación en las aulas de Infantil y Primaria en los centros educativos. 


\section{REFERENCIAS BIBLIOGRÁFICAS}

Aguilar, C. (2013). Género y formación crítica del profesorado: una tarea urgente y pendiente. Revista Interuniversitaria de Formación del Profesorado, 78(27,3), 23-34. Recuperado de http://www.aufop.com/aufop/revistas/arta/impresa/180/1842

Anguita, R. y Torrego, L. (2009). Género, educación y formación del profesorado. Retos y posibilidades. Revista Interuniversitaria de Formación del Profesorado, 64(23,1), 17-25. Recuperado de http://www.aufop.com/aufop/uploaded_files/revistas/1234883102.pdf

Aslan, G. (2015). A Metaphoric Analysis Regarding Gender Perceptions of Preservice Teachers. Education and Science, 40(181), 363-384. doi: 10.15390/EB.2015.2930

Bejarano, M. y Mateos, A. (2014). Género y Sexualidad en la formación inicial de maestros y maestras. ¿Por qué no un currículum sexual? Exedra, Revista científica, número temático, 127-146. Recuperado de http://www.exedrajournal.com/wp-content/uploads/2014/12/sup14-127146.pdf

Benhabib, S. (2011). Dignity in Adversity: Human Rights in Troubled Times. Cambridge/Malden: Polity Press.

Butler, J. (2001). La cuestión de la transformación social. En BeckGernsheim, Elisabeth; Butler, Judith y Puigvert, Lidia. Mujeres y transformaciones sociales (pp.7-30). Barcelona: El Roure Editorial.

Case, K., Hensley, R. y Anderson, A. (2014). Reflecting on Heterosexual and Male Privilege: Interventions to Raise Awareness. Journal of Social Issues, 70(4), 722-740. doi: 10.1111/josi.12088.

Castells, M. (1998). La era de la información (Vol. 2): Economía, Sociedad y cultura. El poder de la identidad. Madrid: Alianza.

Cochran-Smith, M. (2009). Re-culturing teacher education: inquiry evidence and action. Journal of Teacher Education, 60(5), 458-468. doi: $10.1177 / 0022487109347206$.

Comins, I. (2003). Del Miedo a la Diversidad a la Ética del Cuidado: Una Perspectiva de Género. Convergencia, 33, 97-122. Recuperado de http://www.redalyc.org/pdf/105/10503305.pdf

Comins, I. (2008). La ética del cuidado y la construcción de la paz. Barcelona: Icaria.

Donoso-Vázquez, T. y Velasco-Martínez, A. (2013). ¿Por qué una propuesta de formación en perspectiva de género en el ámbito universitario? Profesorado. Revista de currículum y formación del profesorado, 17(1), 7288. Recuperado de http://www.ugr.es/ recfpro/rev171ART5.pdf 
Engebretson, K. E. (2016). Talking (fe)male: examining the gendered discourses of preservice teachers. Gender and Education, 28(1), 37-54, doi.10.1080/09540253.2015.1096917.

Fascioli, A. (2010). Ética del cuidado y ética de la justicia en la teoría moral de Carol Gilligan. Revista Actio, 12, 41-57. Recuperado de http://actio.fhuce.edu.uy/Textos/12/Fascioli12.pdf

Fernández, A. y López, M. C. (2010). La educación en valores desde la carta de la tierra. Por una pedagogía del cuidado. Revista Iberoamericana de Educación, 53(4), 1-19. Recuperado de http://rieoei.org/3310.htm

Fernández, E. y Piedra, J. (2010). Efecto de una formación coeducativa sobre las actitudes hacia la igualdad en el futuro profesorado de Educación Primaria. Cultura, Ciencia y Deporte, 5(15), 151-158. doi: http://dx.doi.org/10.12800/ccd.v5i15.106

Freixas, A., Fuentes-Guerra, M. y Luque, B. (2007). Formación del profesorado y diferencia sexual. Revista Fuentes, 7, 52-64. Recuperado de http://institucional.us.es/revistas/fuente/7/art 3.pdf

García, R., Sala, A., Rodríguez, E. y Sabuco, A. (2013). Formación inicial del profesorado sobre género y coeducación: impactos metacognitivos de la inclusión curricular transversal sobre sexismo y homofobia. Profesorado. Revista de currículum y formación del profesorado, 17(1), 269-287. Recuperado de http://www.ugr.es/ recfpro/rev171COL1.pdf

García-Cano Torrico, M., Gutiérrez Santiuste, E. e Hinojosa Pareja, E. F. (2016). Innovación en la formación del profesorado mediante la contextualización del aprendizaje en el espacio urbano. En P. Membiela, N. Casado y M. I. Cebreiros (eds.). Presente y futuro de la docencia universitaria (pp. 273-277). Orense: Educación Editora.

García-Pérez, R., Rebollo, A., Vega, L., Barragán-Sánchez, R., Buzón, O. y Piedra, J. (2011). El patriarcado no es transparente: Competencias del profesorado para reconocer desigualdad. Cultura y Educación, 23(3), 385397. doi: http://dx.doi.org/10.1174/113564011797330298.

Gilligan, C. (1994 [1986]). La moral y la teoría. Psicología del desarrollo femenino. México: Fondo de Cultura Económica.

Giroux, H. (1990). Los profesores como intelectuales. Hacia una pedagogía crítica del aprendizaje. Madrid: Paidós.

Goldstein, L. S. y Lake, V. E. (2000). "Love, love, and more love for children": exploring preservice teachers' understandings of caring. Teaching and Teacher Education, 16, 861-872. doi: http://dx.doi.org/10.1016/S0742-051X(00)00031-7.

Gozálvez, V. y Jover, G. (2016). Articulación de la justicia y el cuidado en la educación moral: Del universalismo sustitutivo a una ética situada de 
los derechos humanos. Educación XX1, 19(1), 311-330. doi:10.5944/educXX1.14221.

Instituto de la Mujer (2004). Estudio para conocer la situación actual de la educación para la igualdad en España. Madrid: Instituto de la Mujer. Recuperado de http://www.educarenigualdad.org/upload/Doc_15_red2red.pdf

Kohlmeier, J. y Saye, J. W. (2012). Justice or Care? Ethical Reasoning of Preservice Social Studies Teachers. Theory $y$ Research in Social Education, 40(4), 409-435. doi: 10.1080/00933104.2012.724361.

Lagarde, M. (1998). Identidad de género y derechos humanos. La construcción de las humanas. En G. Papadimitriou (Coord.). Educación para la paz y los derechos humanos. Distintas miradas (pp. 71-106). México: Asociación Mexicana para las Naciones Unidas.

Ley Orgánica 3/2007, de 22 de marzo, para la igualdad efectiva de mujeres y hombres. Cortes Generales de España. BOE núm. 171 del 23 de Marzo de 2007.

López, M. C., Hinojosa, E. F. y Sánchez, D. (2014). Evaluación de la calidad de los proyectos de innovación docente universitaria. Profesorado, Revista de currículum y formación del profesorado, 18(3), 377-391. Recuperado de http://www.ugr.es/ recfpro/rev183COL10.pdf

Manzano-Arrondo, V. (2015). Activismo frente a norma: ¿quién salva a la universidad? RIDAS, Revista Iberoamericana de Aprendizaje Servicio, 1, 28-55. doi: http://dx.doi.org/10.1344/RIDAS2015.1.3

Moreno, E. (2007). La transmisión de modelos sexistas en la escuela. En M. A. Santos (Coord.). El harén pedagógico. Perspectiva de género en la organización escolar (pp. 11-30). Barcelona: Graó.

Noddings, N. (2009). La educación moral. Buenos Aires: Amorrortu.

Real Decreto 1393/2007, de 29 de octubre, por el que se establece la ordenación de las enseñanzas universitarias oficiales. BOE núm. 260 del 30 de Octubre de 2007.

Rebollo, M. A. (2013). La innovación educativa con perspectiva de género. Retos y desafíos para el profesorado. Editorial. Profesorado, Revista de currículum y formación del profesorado, 17(3), 3-8. Recuperado de www.ugr.es/ recfpro/rev171ed.pdf

Romero, A. y Abril, F. (2008). Género y la formación del profesorado en los estudios de Educación Infantil. REIFOP, 11(3), 43-51. Recuperado de www.aufop.com/aufop/uploaded files/articulos/1240850050.pdf

Rué, J. (2013). Formación docente del profesorado universitario. Una perspectiva internacional. Revista de Docencia Universitaria, 11(3), 1722. doi: http://dx.doi.org/10.4995/redu.2013.5516. 
Ryan, M. y Ryan, M. (2013). Theorising a model for teaching and assessing reflective learning in Higher Education. Higher Education Research and Development, 32(2), 244-257. doi: 10.1080/07294360.2012.661704.

Subirats, M. (1998). Con diferencia: las mujeres frente al reto de la autonomía. Barcelona: Icaria.

Subirats, M. y Brullet, C. (1990). La coeducación. Madrid: Secretaría de Estado de Educación. Ministerio de Educación y Ciencia.

Torras, M. E. (2013). El aprendizaje cooperativo en línea y la ética del cuidado. Estudios sobre educación, 24, 149-171. Recuperado de https://www.unav.edu/publicaciones/revistas/index.php/estudios-sobreeducacion/article/view/2029/1894

Vázquez, V. y Escámez, J. (2010). La profesión docente y la ética del cuidado. Revista Electrónica de Investigación Educativa [Número Especial]. Recuperado de http://redie.uabc.mx/contenido/NumEsp2/contenidoverdera.html.

Ventura, A. (2009). El procés de Bolonya i els estudis de gènere. Quaderns d'Educació Contínua, 20, 13-19. Recuperado de http://www.raco.cat/index.php/educaciosocial/article/viewFile/165604/2 $\underline{41192}$

Vizcarra, M. T., Nuño, T., Lasarte, G., Aristizabal, P. y Álvarez-Uria, A. (2015). La perspectiva de género en los títulos de Grado en la Escuela Universitaria de Magisterio de Vitoria-Gasteiz. REDU, Revista de Docencia Universitaria, 13(1), 297-318. Recuperado de http://redu.net/redu/index.php/REDU/article/view/713

Walker, M. y McLean, M. (2013). Professional Education, Capabilities and the Public Good: The Role of universities promoting human development. New York: Routledge. 